\title{
Substance Use Start Date Time
}

National Cancer Institute

\section{Source}

National Cancer Institute. Substance Use Start Date Time. NCI Thesaurus. Code C83431.

The date and time substance usage began. 